\title{
Limosilactobacillus reuteri ATCC PTA 5289 and DSM 17938 as adjuvants to improve evolution of pharyngitis/tonsillitis in children: randomised controlled trial
}

\author{
A. Maya-Barrios ${ }^{1}$, K. Lira-Hernandez ${ }^{2}$, I. Jiménez-Escobar ${ }^{1}$, L. Hernández ${ }^{1}$, A. Ortiz-Hernandez ${ }^{3}$, C. Jiménez- \\ Gutiérrez $^{1}$, G. López-Velázquez ${ }^{4}$ and P. Gutiérrez-Castrellón ${ }^{2,5 *}$ \\ ${ }^{1}$ Paediatric's Division, Hospital GEA, Calzada de Tlalpan 4800, 14080 Mexico City, Mexico; ${ }^{2}$ Centre for Translational \\ Research on Mother and Child Health, Hospital GEA, Calzada de Tlalpan 4800, 14080, Mexico City, Mexico; ${ }^{3}$ Emergency \\ Department, Instituto Nacional de Pediatría, Insurgentes sur 3700-C, 04530 Mexico City, Mexico; ${ }^{4}$ Grupo de Investigación \\ en Biomoléculas y Salud Infantil, Lab. EIMyT, Instituto Nacional de Pediatría, Insurgentes sur 3700-C, 04530 Mexico \\ City, Mexico; ${ }^{5}$ Sociedad Latino Americana de Gastroenterología, Hepatologia y Nutrición Pediátrica (LASPGHAN) E \\ International Scientific Council for Probiotics, Tenango 22, 14340, Mexico City, Mexico; programacionmetabolica@gmail.com
}

Received: 10 September 2020 / Accepted: 19 December 2020

(c) 2021 Wageningen Academic Publishers

\section{OPEN ACCESS (c) (1) (2) RESEARCH ARTICLE}

\begin{abstract}
Pharyngitis and tonsillitis are the most common acute respiratory infections (ARIs) in children aged $\leq 5$ years. The analysis of published data showed that some probiotics could decrease the frequency and number of days with ARIs. This study evaluated the safety and efficacy of Limosilactobacillus reuteri ATCC PTA 5289 and DSM 17938 to reduce the duration and severity of ARI symptoms. This randomised controlled trial included children aged from 6 months to 5 years, with pharyngitis or tonsillitis, who were randomised to receive a probiotic product containing L. reuteri ATCC PTA 5289 and L. reuteri DSM 17938 or placebo, as drops, ingested orally for 10 days as adjuvants to the use of non-steroidal anti-inflammatory drugs. The main outcomes were the duration and severity of ARI symptoms. The secondary outcomes were changes in salivary immunoglobulin A and inflammatory biomarkers. There was no fever on day 2 and subsequent days in the L. reuteri group $\left(37.3 \pm 0.5^{\circ} \mathrm{C}\right.$ vs $\left.38.6 \pm 0.3{ }^{\circ} \mathrm{C}, P<0.05\right)$. Beginning on day 3 , the severity of sore throat ( $5 \pm 0.9$ vs $8 \pm 1.2, P<0.05$ ) was lower in the $L$. reuteri group. Significant differences in the days with runny nose, nasal congestion, days of non-programmed visits to the medical office or emergency department, levels in tumoral necrosis factor-alpha (TNF-alpha) and related costs of treatment were observed in the $L$. reuteri group. The frequency of adverse events was similar between the groups. Therefore, $L$. reuteri ATCC PTA 5289 combined with L. reuteri DSM 17938 is a safe and effective adjunct to reduce the symptoms of pharyngitis or tonsillitis in children.
\end{abstract}

Keywords: L. reuteri, respiratory infections, paediatrics.

\section{Introduction}

Acute respiratory infections (ARIs) in children are a public health problem, and independent of the world region, children can have 3-10 episodes annually (Chonmaitree et al., 2008; Monto et al., 1971; Wald et al., 1991). Approximately $85 \%$ of all ARIs are upper respiratory tract infections (URTIs), of which pharyngitis and tonsillitis are the most common (Jain et al., 2001; Peasah et al., 2015; Singh and Nayar, 1996; Tambe et al., 1999). Moreover, $70-80 \%$ of these infections are caused by viruses, with rhinovirus, coronavirus and adenovirus accounting for $40-60 \%$ of the total cases (Bisno, 2001). For bacterial cases, Streptococcus Group A (GABHS) represents one of the most important infectious agents (Shaikh et al., 2010). In most cases, the management of URTIs is ambulatory, and infections are associated with remarkable direct and indirect costs and significant deterioration of quality of life (Peasah et al., 2015). Unfortunately, although ARIs are generally self-limiting, and $>70 \%$ are of viral aetiology, the use of antibiotics is standardised worldwide. For example, between 33 and $75 \%$ of children and adolescents aged $<15$ 
years are receiving antibiotic therapy because of URTIs in Germany (Holstiege and Garbie, 2013). This inappropriate antibiotic use is usually associated with bacterial resistance, disturbance of normal balance of human microbiota and colonisation of pathogens (Andrews et al., 2012; Lange et al., 2016). Practice guidelines differ considerably regarding how to initiate treatment. Some guidelines consider that bacterial URTIs, especially GABHS, must be treated with antibiotics to avoid complications (AAP, 2006; AFSS, 2003; Bisno et al., 2002; Gerber et al., 2009; NGCH, 2001; Snow et al., 2001), while others consider that even GABHS is a benign self-limiting disease and recommend the use of antibiotics only in selected cases to prevent resistance (De Meyere and Matthys, 1999; NICE, 2008; SIGN, 2010; Starreveld et al., 2008). A cohort study conducted in primary care settings with $>3$ million episodes of ARIs identified that the number needed to treat for antibiotics to prevent one complication (e.g. otitis media) was 4,000, concluding that it is not cost-effective to use antibiotics in all cases of URTIs (Petersen et al., 2007).

The use of probiotics as an adjuvant for treatment or prevention of URTIs in children has been evaluated in several studies, and since 2001, approximately 45 randomised controlled trials (RCTs) have been published. Recently, Wang et al. (2016) conducted a systematic review and meta-analysis that aimed to evaluate the efficacy of probiotics as adjuvants in children with URTIs. The analysis showed that probiotic consumption significantly decreased the number of subjects with at least one respiratory tract infection episode (relative risk, 0.89; 95\% confidence interval [CI], 0.82-0.96; $P=0.004)$, numbers of days of URTIs per child (mean differences [MD], -0.16; 95\% CI, -0.29-0.02; $P=0.03$ ) and days absent from day care/school (MD, -0.94; 95\% CI, -1.72 to $-0.15 ; P=0.02$ ) (Wang et al., 2016). Several studies have shown the positive effect of Limosilactobacillus (Lactobacillus) reuteri for prevention of URTIs in children (Agustina et al., 2012; Gutierrez-Castrellon et al., 2014; Weizman et al., 2005). Weizman et al. (2005) published a double-blind, placebo-controlled, randomised trial conducted in child care centres in Israel in children aged 4-10 months old. L. reuteri ATCC 55730, Bifidobacterium lactis BB-12 or placebo was administrated to 68,73 and 60 children, respectively, reporting that children who received $L$. reuteri had less respiratory infections episodes (Weizman et al., 2005). Agustina et al. (2012) published an randomised controlled trial (RCT) in healthy children aged 1-6 years who received Lacticaseibacillus (Lactobacillus) casei CRL431 ( $\mathrm{n}=120)$ or L. reuteri DSM $17938(\mathrm{n}=124)$. The study did not show any significant effect on URTIs (Agustina et al., 2012). An RCT on healthy children, 6-36 months, attending day care centres where the subjects received $L$. reuteri DSM $17938,10^{8} \mathrm{cfu}(\mathrm{n}=168)$, or placebo ( $\mathrm{n}=168$ ), for 3 months, with an additional 3 months followup without supplementation, was performed by our group. The study revealed that $L$. reuteri DSM 17938 significantly reduced the frequency and duration of respiratory tract infections at both 3 and 6 months $(P<0.05)$. Additionally, the number of physician visits, antibiotic use, absenteeism from day care centres and parental absenteeism from work were significantly reduced in the $L$. reuteri Group $(P<0.05)$. A cost-benefit analysis revealed significant reductions in costs in the $L$. reuteri-supplemented children. No adverse events related to the study product were reported (GutierrezCastrellon et al., 2014).

The present study aimed to evaluate the safety and efficacy of L. reuteri ATCC PTA 5289 combined with L. reuteri DSM 17938 , as an adjuvant to non-steroidal anti-inflammatory drug (NSAID), twice daily for 10 days in children with URTIs aged 6 months to 5 years. The $L$. reuteri strains DSM 17938 and ATCC PTA 5289 were selected due to their antiviral and antimicrobial properties and their effects on the immune system and mucosal integrity. Both strains have the ability to produce reuterin, a substance with both antiviral and antimicrobial activity (Ang et al., 2016; Spinler et al., 2008). Interestingly, the strains also produce reuterin when growing in biofilms. Strain ATCC PTA 5289 is more capable than the mother strain of DSM 17938, ATCC 55730, to form biofilms (Jones and Versalovic, 2009; Rosander et al., 2008). L. reuteri strains have also been reported to increase the concentration of secretory immunoglobulin (Ig)A (Mu et al., 2018), which could be an important factor in the defence against pathogens. Finally, different strains of $L$. reuteri have been shown to protect integrity of cultivated intestinal epithelial cells from the detrimental effect of enterotoxigenic Escherichia coli by increasing the expression of the longer isoform of zonulin (ZO)-1, maintaining E-cadherin expression and downregulating induction of interleukin (IL)-6 and umour necrosis factor (TNF)- $\alpha$ (Karimi et al., 2018).

\section{Material and methods}

\section{Study design, ethics and informed consent}

This randomised, double-blind, placebo-controlled trial was approved by the Research and Ethical Research Committees at Hospital General Dr. Manuel Gea Gonzalez, ID 39-141-2017 and registered at ClinicalsTrials.gov ID NCT03377374. Each parent or legal tutor has signed an informed consent form before infants were enrolled in this study. Participants were recruited in Child Care Centre and Paediatric Emergency Department at Hospital General Dr. Manuel Gea González, Ministry of Health, México.

\section{Participant selection}

Children aged from 6 months to 5 years of both sexes, born at term with a birth weight $>2,500 \mathrm{~g}$ and a similar socioeconomic background, were eligible if they met the study definition of pharyngitis and/or tonsillitis. Pharyngitis 
was defined as presence of sore throat, fever and pain during swallowing. At visualisation, the pharynx shows swelling and redness and covered with a thick mucus material. Tonsillitis was defined as the presence of swelling or redness of tonsils with discharge, fever and pain during swallowing.

Children were excluded if they had recurrent ARIs (eight or more new episodes of otitis media, two or more serious sinus infections or two or more episodes of pneumonia within 12 months); history of two or more invasive infections (meningitis, cellulitis, osteomyelitis, septicaemia), failure to thrive, chronic diarrhoea; recurrent deep skin or organ abscesses; persistent superficial candidiasis after 1 year of age; use of antibiotics for respiratory infections for two or more months in the past 12 months; gastroesophageal reflux; atopic disease (allergic rhinitis or asthma); recent (within the preceding 4 weeks) exposure to probiotics, prebiotics or antibiotics and supplementation of probiotics 2 weeks before inclusion in the study and during the study period.

\section{Research outcomes and sample size}

The primary outcome was duration of upper respiratory symptoms \& severity of sore throat. The Face, Legs, Activity, Cry, Consolability (FLACC) assessment tool provided a score for evaluation of severity of pain (sore throat), through facial expressions, leg movements, activity, crying pattern and level of consolability with a minimum score of 0 and a maximum of 10 (Merkel et al., 1997). Secondary outcomes were frequency of rhinorrhea, cough episodes, nasal congestion, snoring episodes, sleep disturbances, days with fever (temperature $>37.5^{\circ} \mathrm{C}$, measured four times daily, using standardised thermometers and highest value during the day reported); number of children receiving antibiotic treatment; total number of days for antibiotic use [antibiotic needs were evaluated using the Pragmatic Scoring System for Pharyngitis, considering age of children, presence of tender cervical nodes, signs of headache, petechia on the palate, abdominal pain, sudden onset, conjunctivitis, coryza or diarrhoea, with a score $>3$ requiring antibiotics (Joachim et al., 2010)]; number of visits to medical office or emergency department; days of absence from day care centre; frequency of adverse events; aetiology of respiratory infection and change in salivary inflammatory biomarkers [inflammatory response: $\mathrm{C}$-reactive protein (CRP), IL-1b, IL-8, IL- 6 and TNF- $\alpha$; anti-inflammatory response: $\operatorname{sIgA}$ and IL-10 and immune modulation response: soluble tolllike receptor (sTLR)-2, sTLR4 and sCD14]. Compliance was measured by requesting the parents to return the investigational product at day 10 after randomisation and counting the remaining drops. Considering the primary outcome with a difference between groups of at least $24 \pm 8$ $\mathrm{h}$ on duration of symptoms, and alfa error of $5 \%$, a power of $80 \%$ and using Stata SE version 16.0 (StataCorp LLC,
College Station, TX, USA) a minimum sample size of 30+5 children (losses to follow-up) was calculated.

\section{Interventions}

L. reuteri (DSM 17938 and ATCC PTA 5289) oil drops were administered orally in a total daily dose of $4 \times 10^{8}$ cfu for 10 days. Five drops, containing $2 \times 10^{8} \mathrm{cfu}$, were obtained twice daily (in the morning and evening). The active study product consisted of freeze-dried L. reuteri suspended in a mixture of pharmaceutical grade medium chain triglyceride and sunflower oils. The placebo drops had identical ingredients, except that they lack bacteria. The investigational products, identical in taste and packaging, were supplied by BioGaia AB (Stockholm, Sweden). To guarantee the viability of the live bacteria, the investigational products were kept refrigerated $\left(+2\right.$ to $\left.8^{\circ} \mathrm{C}\right)$ during the study period. Viability analysis showed that the strains in oil were stable. Batch 7DTW117 and batch 6DTW256, used to prepare oil drops for this trial, showed concentrations (cfu/5 drops) of $7.0 \times 10^{8}, 7.9 \times 10^{8}, 7.0 \times 10^{8}, 4.07 \times 10^{8}$ and $6.97 \times 10^{8}$ at 3, 6, 12, 18 and 21 months, respectively. Each child was randomly allocated to the probiotic or control group following a centralised, randomisation procedure.

\section{Study procedures}

After parents signed the informed consent, children were randomised using balanced blocking technique in the probiotic or placebo group. Blinding and randomisation were performed by the sponsor, BioGaia AB (Stockholm Sweden), using the website randomisation.com (http:// www.randomization.com). Demographic data, medical history and physical examination, including vital signs and anthropometrics, were obtained from all eligible participants at baseline visit. Nasopharyngeal samples were obtained using nylon-specific respiratory swabs (Copan Diagnostics) to identify aetiology using Luminex (Abbott Diagnostics, Abbott Park, MI, USA). A saliva sample was obtained and prepared within $1 \mathrm{~h}$ of collection. Protease inhibitor cocktail (Sigma Aldrich, St. Louis, MO, USA), 2 $\mu \mathrm{l} / \mathrm{ml}$ of saliva and sodium orthovanadate $(400 \mathrm{mM}$, Sigma Aldrich) and $3 \mu \mathrm{l} / \mathrm{ml}$ of saliva were added to each sample, which was then centrifuged at $4{ }^{\circ} \mathrm{C}$ and $1,500 \times g$ for 15 $\mathrm{min}$. Then, saliva samples were divided into aliquots and frozen at $-80{ }^{\circ} \mathrm{C}$ until assayed. SIgA levels were analysed as previously described (López-Velázquez et al., 2015), and salivary IL-1b, IL-8, IL-6, CRP, TNF- $\alpha$, IL-10, sTLR2, sTLR4 and sCD14 were measured by ELISA. The parents or legal guardian were trained to fill out a daily diary to record the symptoms during the study period. During the treatment period, every $48 \mathrm{~h}$, a designated investigator called the parents to check for progression, presence of any adverse events or other information important for the study. Ten days after randomisation, physical examination, including vital signs and anthropometrics, was performed. 
A second salivary sample to evaluate sIgA and inflammatory biomarkers was obtained. When the decision, based on the Pragmatic Scoring System for Pharyngitis, was to start antibiotic treatment, the first option was amoxicillin + clavulanate $45 \mathrm{mg} / \mathrm{kg} /$ day BID for seven days. In subjects with a history of allergy to penicillin, the second choice was clarithromycin $7.5 \mathrm{mg} / \mathrm{kg} /$ day twice daily for seven days. The use of antibiotics was recorded in the daily diary and electronic case report form (e-CRF). Costs/child (USD) related to visits to emergency department or ambulatory office secondary to upper respiratory episodes during the study, and costs related to the use of anti-inflammatory drugs, antipyretic, or antibiotics where summarised and included on the outcome analysis for each group.

\section{Statistical analyses}

The intention-to-treat (ITT) statistical approach was used. Randomised subjects who fulfilled the inclusion criteria and none of the exclusion criteria that had received at least 3 days of the investigational product and from whom post randomisation data for the primary endpoints were available were included in the ITT analysis. The duration of exposure to the investigational product and compliance were summarised per group. Changes in duration and severity of respiratory symptoms, duration of fever, use of antibiotics, number of visits to the medical office, number of visits to emergency department, number of days of absence from day care centres and costs related were compared through t-test. The frequency of adverse events in each group was compared using chi-square or Fisher test. IgA levels in the saliva and levels of salivary inflammatory biomarkers were compared between groups through t-test. The frequency of identified viral or bacterial agents in each group were compared using chi-square or Fisher test. In all sets of hypothesis analysis, differences were considered significant if the $P$-value $<0.05$. Stata 15.0 for Mac was used to perform all statistical analyses.

\section{Results}

Seventy children, randomised to receive L. reuteri $(\mathrm{n}=35)$ or placebo $(n=35)$, were included in the study (Figure 1$)$. The number of boys and girls was similar between the groups. The age of the children was $2.1 \pm 0.8$ years in the L. reuteri group and 2.4 \pm 1.1 years in the placebo group $(P$-value not significant). No statistically significant differences were observed when children were included in the study, in relation to the number of days of symptoms prior to study entry, duration of fever and severity of sore throat (FLACC score), symptoms observed, use of anti-inflammatory drugs and levels of sIgA and inflammatory markers (Table 1).

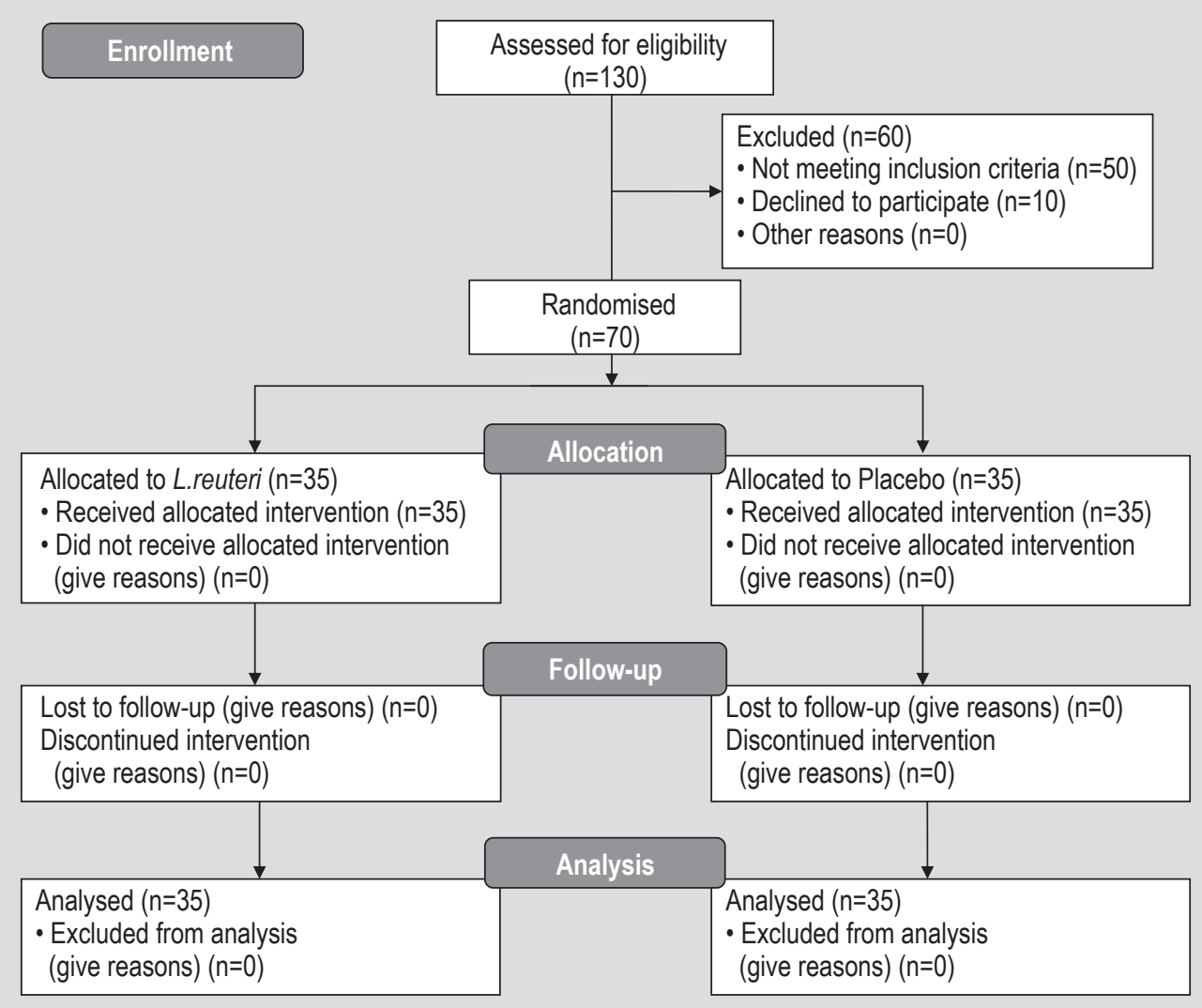

Figure 1. CONSORT flow diagram. 
Table 1. Baseline characteristics at study entry.

\begin{tabular}{|c|c|c|}
\hline Parameter $^{1}$ & Limosilactobacillus reuteri group $(\mathrm{n}=35$ ) & Placebo group $(n=35)$ \\
\hline Age in years (mean $\pm S D$ ) & $2.1 \pm 0.8$ & $2.4 \pm 1.1$ \\
\hline Number of girls $[n(\%)]$ & $19(55)$ & $18(52)$ \\
\hline Days with respiratory symptoms before recruitment (mean \pm SD & $0.8 \pm 0.2$ & $1.0 \pm 0.3$ \\
\hline Days with fever (mean $\pm S D)$ & $0.7 \pm 0.4$ & $0.9 \pm 0.6$ \\
\hline Severity of sore throat (FLACC score) (mean $\pm S D$ ) & $8 \pm 5$ & $9 \pm 4$ \\
\hline Rhinorrhea $[n(\%)]$ & $25(71)$ & $24(68)$ \\
\hline Cough episodes [n (\%)] & $20(58)$ & $21(60)$ \\
\hline Nasal congestion [n (\%)] & $11(32)$ & $10(29)$ \\
\hline Snoring episodes [n (\%)] & $10(29)$ & $11(32)$ \\
\hline Sleep disturbances [n (\%)] & $11(32)$ & $10(29)$ \\
\hline Use of NSAIDs [n (\%)] & $31(88)$ & $29(83)$ \\
\hline \multicolumn{3}{|l|}{ Salivary biomarkers (mean $\pm S D$ ) } \\
\hline $\operatorname{lgA}(\mathrm{mg} / \mathrm{ml})$ & $13.2 \pm 8.1$ & $12.9 \pm 10.3$ \\
\hline IL-1beta (pg/ml) & $42 \pm 12.1$ & $38 \pm 9.9$ \\
\hline IL-8 (pg/ml) & $223 \pm 93$ & $211 \pm 78$ \\
\hline IL-6 (pg/ml) & $8.4 \pm 1.2$ & $9.2 \pm 1.8$ \\
\hline C-reactive protein (mg/l) & $1.22 \pm 0.44$ & $1.30 \pm 0.52$ \\
\hline TNF-a (pg/ml) & $7.2 \pm 1.3$ & $6.9 \pm 0.8$ \\
\hline IL-10 (pg/ml) & $5.5 \pm 2.1$ & $5.8 \pm 1.9$ \\
\hline sTLR2 (pg/ml) & $330 \pm 30$ & $282 \pm 90$ \\
\hline sTLR4 (pg/ml) & $1,005 \pm 280$ & $998 \pm 116$ \\
\hline $\mathrm{sCD} 14(\mathrm{pg} / \mathrm{ml})$ & $2,000 \pm 305$ & $1,892 \pm 224$ \\
\hline
\end{tabular}

The aetiology of pharyngitis or tonsillitis was also similar between the groups (Table 2). At the end of the study, significant differences were observed in relation to the duration of respiratory symptoms $(3.1 \pm 0.8$ vs $5.2 \pm 1.4$, $P<0.05)$, days with fever $(1.8 \pm 0.4$ vs $3.6 \pm 0.6, P<0.05)$, severity of sore throat (FLACC score $5 \pm 1.2$ vs $8 \pm 3.4, P<0.05$ ), runny nose, nasal congestion, days of non-programmed visits to medical office or emergency department and related costs in favour to L. reuteri group.
There was no fever on day 2 and subsequent days in the $L$. reuteri droup ( $37.3 \pm 0.5$ vs $38.6 \pm 0.3^{\circ} \mathrm{C}, P<0.05$ ) (Figures 2 and 3). Significant differences were also identified for TNF- $\alpha$ in favour to the $L$. reuteri Group ( $3.8 \pm 0.7$ vs $5.9 \pm 0.4$ $\mathrm{pg} / \mathrm{ml}, P<0.05$ ) (Table 3 ).

The frequency of adverse events was similar between the groups (Table 4). Compliance to use the investigational

\section{Table 2. Aetiology for upper respiratory infections [n (\%)]. ${ }^{1}$}

\section{Microorganism}

Rhinovirus

Adenovirus

Influenza $A$

Influenza $B$

Syncytial respiratory virus

Coronavirus

Moraxella catarrhalis

Streptococcus pneumoniae

\section{Limosilactobacillus reuteri group ( $n=35) \quad$ Placebo group $(n=35)$}

$\begin{array}{cc}30(11) & 32(11) \\ 28(10) & 31(10) \\ 12(4) & 16(6) \\ 18(6) & 12(4) \\ 7(2) & 6(2) \\ 3(1) & 2(1) \\ 2(1) & 3(1) \\ 6(2) & 4(2)\end{array}$

${ }^{1}$ Figures are not $100 \%$ because there were mixed infections. 


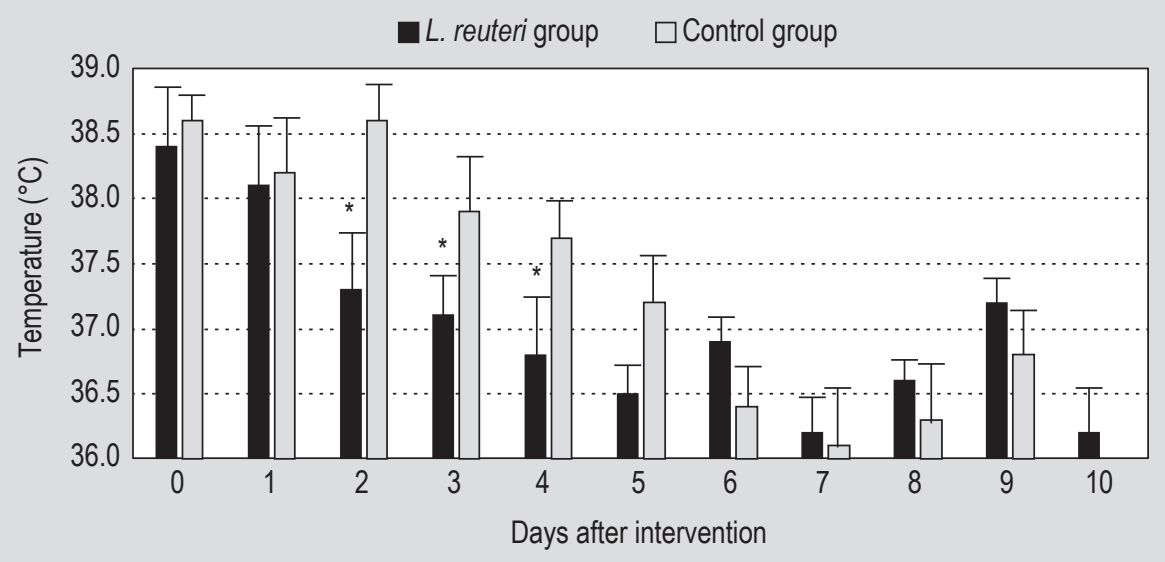

Figure 2. Limosilactobacillus reuteri and evolution of fever in children with pharyngitis/tonsillitis. ${ }^{*} P<0.05$

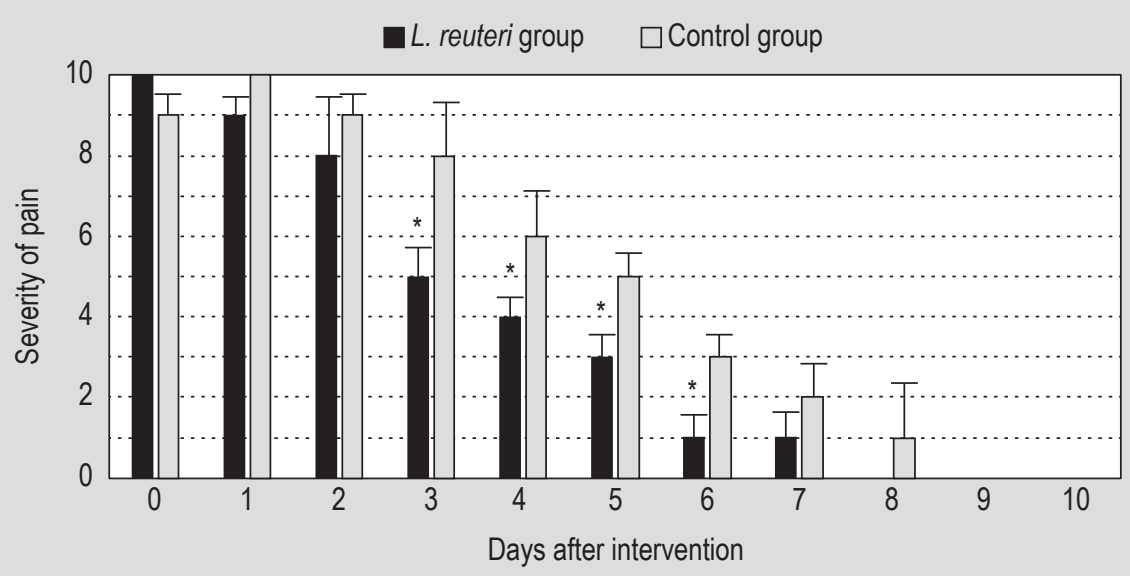

Figure 3. Face, legs, activity, cry, consolability (FLACC) score in children with pharyngitis/tonsillitis. ${ }^{*} P<0.05$

products was $98 \%$ and $97 \%$ for the L. reuteri and placebo groups, respectively.

\section{Discussion and conclusions}

URI is the most frequent acute infection in children aged $<5$ years, with a significant impact on public health worldwide and a significant economic burden on the patients with the disease. The frequency of URTIs is significantly increased in children who attend day care centres due to the greater burden of pathogens they are in contact with (Thrane et al., 2001). A significant associated problem is related to the use of antibiotics even though most of these diseases are caused by viruses, as observed in our study and in correlation with what have been observed in other studies. Although we did not observe significant differences in the use of antibiotics in our study, the use of probiotics in this study, could reduce the use of antibiotics and therefore decrease the high frequency of adverse events observed due to their inappropriate use. In the control group of children in our study, we observed the persistence of fever and pharyngeal pain until the fifth day of supplementation. This type of behaviour usually moves the parents to look for therapeutic alternatives that could contribute to improve symptoms, such as fever, pain and inflammation. Recently, a systematic review with meta-analysis was published, with the aim of evaluating the efficacy of different specific strains of probiotics as adjuvants in the treatment of URTIs in children attending day care centres. The analysis of 12 clinical trials with $>4,500$ children showed that some specific strains significantly reduced the duration of URTIs (Pilmann-Laursen and Hojsak, 2018). Our proof-of-concept (PoC) RCT showed that the product with the combination of two strains of L. reuteri (ATCC PTA 5289 and DSM 17938) reduced not only the duration of days of fever and pharyngeal pain but also other respiratory symptoms, such as rhinorrhoea and nasal congestion, which results in extreme discomfort in young children. The importance of this PoC lies not only on the clinical impact observed but also on the reduction of related costs, which undoubtedly 
Table 3. Primary and secondary outcome analysis at day 10.

\begin{tabular}{|c|c|c|}
\hline Parameter ${ }^{1}$ & Limosilactobacillus reuteri group $(\mathrm{n}=35)$ & Placebo group $(n=35)^{2}$ \\
\hline \multicolumn{3}{|l|}{ Primary outcomes } \\
\hline Duration of symptoms (days) (mean \pm SD) & $3.1 \pm 0.8$ & $5.2 \pm 1.4^{*}$ \\
\hline Severity of sore throat (FLACC Score) (mean \pm SD) & $5.0 \pm 1.2$ & $8.0 \pm 3.4^{*}$ \\
\hline \multicolumn{3}{|l|}{ Secondary outcomes } \\
\hline Rhinorrhea [n (\%)] & $6(17)$ & $15(43)^{*}$ \\
\hline Cough episodes [n (\%)] & $6(17)$ & $7(21)$ \\
\hline Nasal congestion [n (\%)] & $3(8)$ & $10(28)^{*}$ \\
\hline Snoring episodes [n (\%)] & $4(12)$ & $5(14)$ \\
\hline Sleep disturbances [n (\%)] & $3(8)$ & $4(11)$ \\
\hline Days with fever (mean \pm SD) & $1.8 \pm 0.4$ & $3.6 \pm 0.6^{*}$ \\
\hline Use of antibiotics [n (\%)] & $2(6)$ & $4(12)$ \\
\hline $\begin{array}{l}\text { Number of non-programmed visits to medical office or emergency } \\
\text { department (mean } \pm S D)\end{array}$ & $0.8 \pm 1.1$ & $1.4 \pm 1.2^{*}$ \\
\hline Days of absence to day care centre (mean $\pm S D$ ) & $3.2 \pm 0.9$ & $2.9 \pm 1.1$ \\
\hline Average cost/child (USD\$) (mean $\pm S D)$ & $71 \pm 10$ & $101 \pm 19^{*}$ \\
\hline TNF- $a(p g / m l)(m e a n \pm S D)$ & $3.8 \pm 0.7$ & $5.9 \pm 0.4^{*}$ \\
\hline
\end{tabular}

Table 4. Frequency of adverse events divided by age groups.

$\begin{array}{llll}\text { Adverse event } & & \text { Limosilactobacillus reuteri group } & \text { Placebo group } \\ \text { 6-12 months }(n=28) & \text { Worsening of rhinorrhoea [n (\%)] } & 7(25) & 6(21) \\ & \text { Change of stool consistency [n (\%)] } & 3(11) & 4(14) \\ & \text { Abdominal pain [n (\%)] } & 1(3) & 2(7) \\ 1-3 \text { years }(n=42) & \text { Worsening of rhinorrhoea [n (\%)] } & 5(12) & 4(9) \\ & \text { Change of stool consistency [n (\%)] } & 1(2) & 2(5) \\ & \text { Abdominal pain [n (\%)] } & 7(17) & 8(19)\end{array}$

adds a significant value from a public health point of view, considering the frequency of ARIs observed in children aged $<5$ years and the impact on the quality of life. In 2014, our research group demonstrated in a similar study conducted in day care centres that the administration of one of the strains (L. reuteri DSM 17938) represented a cost-effective intervention by reducing the expenses related to the integral care of these infections (Gutierrez-Castrellon et al., 2014). In this study, we demonstrate that the use of this combination of $L$. reuteri strains can represent a cost-effectiveness strategy. One of this studies strengths is that we analysed not only the clinical manifestations and etiological profile of the URTIs but also the immunomodulatory profiles of this type of adjuvant. Within the limitations is the fact that this study is a PoC with a small sample size, which limits the extrapolability of the results to other populations. Although we have identified significant differences between the treatment groups, strong enough to establish some recommendations, it is advisable to corroborate the identified findings in a second clinical trial, with a larger sample size, which allows evaluation of the impact of some covariates that could generate different impacts on the outcome variables. Based on our results, we conclude that L. reuteri ATCC PTA 5289 combined with L. reuteri DSM 17938 is an adjuvant to reduce the duration and severity of clinical symptoms in children with pharyngitis or tonsillitis. Due to its anti-inflammatory effects, this combination of strains reduces the duration of fever and intensity of sore throat.

\section{Conflict of interests}

All authors have no potential conflicts of interest to disclose. 


\section{References}

Agence Française de Sécurité Sanitaire des Produits de Santé Clin (AFSS), 2003. Systemic antibiotic treatment in upper and lower respiratory tract infections: official French guidelines. Clinical Microbiology and Infection 9: 1162-1178.

Agustina, R., Kok, F.J., van de Rest, O., Fahmida, U., Lukito, W., Feskens, M.E.J., Van den Heuvel, E.G.H.M., Alber, R. and Bovee-Oudenhoven, I.M.J., 2012. Randomized trial of probiotics and calcium on diarrhoea and respiratory tract infections in Indonesian children. Pediatrics 129: e1155-64. https://doi.org/10.1542/peds.2011-1379

American Academy of Paediatrics (AAP), 2006. Red book. Report of the Committee on Infectious Diseases. Committee on Infectious Diseases, Elk Grove Village, IL, USA.

Andrews, T., Thompson, M., Buckley, D.I., Buckley, D.I., Heneghan, C., Deyo, R., Redmond, N., Lucas, P.J., Blair, P.S. and Hay, A.D., 2012. Interventions to influence consulting and antibiotic use for acute respiratory tract infections in children: a systematic review and meta-analysis. PLoS ONE 7: e30334. https://doi.org/10.1371/ journal.pone.0030334

Ang, L.Y., Too, H.K., Tan, E.L., Chow, T.K., Shek, L.P., Tham, E.H. and Alonso, S., 2016. Antiviral activity of Lactobacillus reuteri protectis against Coxsackievirus A and Enterovirus 71 infection in human skeletal muscle and colon cell lines. Virology Journal 13: 111-112. https://doi.org/10.1186/s12985-016-0567-6

Bisno, A.L., 2001. Acute pharyngitis. New England Journal of Medicine 344: 205-211. https://doi.org/10.1056/NEJM200101183440308

Bisno, A.L., Gerber, M.A., Gwaltney, J.M. Jr., Kaplan, E.L., Schwartz, R.H. and the Infectious Diseases Society of America, 2002. Practice guidelines for the diagnosis and management of group A streptococcal pharyngitis. Clinical Infectious Diseases 35: 113-125. https://doi.org/10.1086/340949

Chonmaitree, T., Revai, K., Grady, J.J., Clos, A., Patel, J.A., Nair, S., Fan, J. and Henrickson, K.J., 2008. Viral upper respiratory tract infection and otitis media complication in young children. Clinical Infectious Diseases 46: 815-823. https://doi.org/10.1086/528685

De Meyere, M. and Matthys, J., 1999. Guideline acute sore throat. Huisarts Nu 2: 193-201. [in Dutch].

Gerber, M.A., Baltimore, R.S., Eaton, C.B., Gewitz, M., Rowley, A.H., Shulman, S.T. and Taubert, K.A., 2009. Prevention of rheumatic fever and diagnosis and treatment of acute streptococcal pharyngitis: a scientific statement from the American Heart Association endorsed by the American Academy of Pediatrics. Circulation 119: 15411551. https://doi.org/10.1161/CIRCULATIONAHA.109.191959

Gutierrez-Castrellon, P., Lopez-Velazquez, G., Diaz-Garcia, L., JimenezGutierrez, C., Mancilla-Ramirez, J., Estevez-Jimenez, J. and Parra, M., 2014. Diarrhea in preschool children and Lactobacillus reuteri: a randomized controlled trial. Pediatrics 133: e904-9.

Holstiege, J. and Garbe, E., 2013. Systemic antibiotic use among children and adolescents in Germany: a population-based study. European Journal of Pediatrics 172: 787-795. https://doi. org/10.1542/peds.2013-0652

Jain, N., Lodha, R. and Kabra, S.K., 2001. Upper respiratory tract infections. Indian Journal of Pediatrics 68:1135-1138. https://doi. org/10.1007/BF02722930.
Joachim, L., Campos, D. Jr. and Smeesters, P.R., 2010. Pragmatic scoring system for pharyngitis in low-resource settings. Pediatrics 126: e608-e614. https://doi.org/10.1542/peds.2010-0569

Jones, S.E. and Versalovic, J., 2009. Probiotic Lactobacillus reuteri biofilms produce antimicrobial and anti-inflammatory factors. BMC Microbiology 9: 35. http://doi.org/10.1186/1471-2180-9-35

Karimi, S., Jonsson, H., Lundh, T. and Roos, S., 2018. Lactobacillus reuteri strains protect epithelial barrier integrity of IPEC-J2 monolayers from the detrimental effect of enterotoxigenic Escherichia coli. Physiological Reports 6: 1351. http://doi. org/10.14814/phy2.13514

Lange, K., Buerger, M. and Stallmach, A., 2016. Effects of antibiotics on gut microbiota. Digestive Diseases 34: 260-268. https://doi. org/10.1159/000443360

López-Velázquez, G., Parra-Ortiz, M., Mora, I.D.l.M.-D.l., GarcíaTorres, I., Enríquez-Flores, S., Alcántara-Ortigoza, M.A., Angel, A.G.-d., Velázquez-Aragón, J., Ortiz-Hernández, R., Cruz-Rubio, J.M., Villa-Barragán, P., Jiménez-Gutiérrez, C. and GutiérrezCastrellón, P., 2015. Effects of fructans from Mexican agave in newborns fed with infant formula: a randomized controlled trial. Nutrients 7: 8939-8951. https://doi.org/10.3390/nu7115442

Merkel, S., Voepel-Lewis, T. and Shayevitz, J.R., 1997. The FLACC: a behavioural scale for scoring postoperative pain in young children. Paediatric Nursing 23: 293-797.

Monto, A.S., Napier, J.A. and Metzner, H.L., 1971. The Tecumseh study of respiratory illness. I. Plan of study and observations on syndromes of acute respiratory disease. American Journal of Epidemiology 94: 269-279. https://doi.org/10.1093/oxfordjournals.aje.a121320

$\mathrm{Mu}$, Q., Tavellav, V.J. and Luo, X.M., 2018. Role of Lactobacillus reuteri in human health and diseases. Frontiers in Microbiology 9: 25. http://doi.org/10.3389/fmicb.2018.00757

National Guideline Clearing House (NGCH), 2001. Sore throat and tonsillitis. Available at: http://www.guideline.gov.

National Institute for Health and Clinical Excellence (NICE), 2008. Prescribing of antibiotics for self-limiting respiratory tract infections in adults and children in primary car (Clinical guideline 69). NICE, London, UK. Available at: http://www.nice.org.uk

Peasah, S.K., Purakayastha, D.R., Koul, P.A., Dawood, F.S., Saha, S., Amarchand, R., Broor, S., Rastogi, V., Assad, R., Kaul, K.A., Widdowson, M.A., Lal, R.B. and Krishnan, A., 2015. The cost of acute respiratory infections in Northern India: a multi-site study. BMC Public Health 15: 330. https://doi.org/10.1186/s12889-0151685-6

Petersen, I., Johnson, A.M., Islam, A., Duckworth, G., Livermore, D.M. and Hayward, A.C., 2007. Protective effect of antibiotics against serious complications of common respiratory tract infections: retrospective cohort study with the UK General Practice Research Database. British Medical Journal 335: 982.

Pilmann-Laursen, R. and Hojsak, I., 2018. Probiotics for respiratory tract infections in children attending day care centre. a systematic review. European Journal of Pediatrics 177: 979-994. https://doi. org/10.1007/s00431-018-3167-1 
Rosander, A., Connolly, E. and Roos, S., 2008. Removal of antibiotic resistance plasmids from Lactobacillus reuteri ATCC 55730 and characterization of the resulting daughter strain L. reuteri DSM 17938. Applied Enviromental Microbiololgy 74: 6032-6040. https:// doi.org/10.1128/AEM.00991-08

Scottish Intercollegiate Guidelines Network (SIGN), 2019. Management of sore throat. Available at: http://www.sign.ac.uk.

Shaikh, N., Leonard, E. and Martin, J.M., 2010. Prevalence of streptococcal pharyngitis and streptococcal carriage in children: a meta-analysis. Pediatrics 126: e557-64.

Singh, M.P. and Nayar, S., 1996. Magnitude of acute respiratory infections in fewer than five children. Journal of Communicable Diseases 28: 273-278.

Snow, V., Mottur-Pilson, C., Cooper, R.J. and Hoffman, J.R. for the American College of Physicians-American Society of Internal Medicine, 2001. Principles of appropriate antibiotic use pharyngitis in adult. Annals of Internal Medicine 134: 506-508. https://doi. org/10.7326/0003-4819-134-6-200103200-00018

Spinler, J.K.J., Taweechotipatr, M.M., Rognerud, C.L.C., Ou, C.N.C., Tumwasorn, S.S. and Versalovic, J.J., 2008. Human-derived probiotic Lactobacillus reuteri demonstrate antimicrobial activities targeting diverse enteric bacterial pathogens. Anaerobe 14: 166-171. https:// doi.org/10.1016/j.anaerobe.2008.02.001
Starreveld, J.S., Zwart, S. and Boukes, F.S., 2008. Summary of the practice guideline 'Sore throat' (second revision) from the Dutch College of General Practitioners. Nederlands Tijdschrift voor Geneeskunde 152: 431-435.

Tambe, M.P., Shivaram, C. and Chandrashekhar, Y., 1999. Acute respiratory infection in children: a survey in the rural community. Indian Journal of Medical Sciences 53: 249-253.

Thrane, N., Olesen, C., Mortensen, J.T., Søndergaard, C., Schønheyder, H.C. and Sørensen, H.T., 2001. Influence of day care attendance on the use of systemic antibiotics in 0- to 2-year-old children. Pediatrics 107: E76. https://doi.org/10.1542/peds.107.5.e76

Wald, E.R., Guerra, N. and Byers, C., 1991. Upper respiratory tract infections in young children: duration of and frequency of complications. Pediatrics 87: 129-133.

Wang, Y., Li, X., Ge, T., Xiao, Y., Liao, Y., Cui, Y., Zhang, Y., Ho, W., Yu, G. and Zhang, T., 2016. Probiotics for prevention and treatment of respiratory tract infections in children: a systematic review and meta-analysis of randomised controlled trials. Medicine 95: e4509. https://doi.org/10.1097/MD.0000000000004509

Weizman, Z., Asli, G. and Alsheikh, A., 2005. Effect of a probiotic infant formula on infections in child care centres: comparison of two probiotic agents. Pediatrics 115: 5-9. https://doi.org/10.1542/ peds.2004-0845 
